\title{
Selective and non-selective cyclooxygenase inhibitors delay stress fracture healing in the rat ulna
}

Kidd LJ ${ }^{a}$, Cowling ${ }^{a}$ NR, Wu AC ${ }^{b}, K^{2}{ }^{b}$ WL, and Forwood MR ${ }^{b}$

${ }^{\mathrm{a}}$ School of Veterinary Science, The University of Queensland, Gatton QLD 4343, Australia.

${ }^{\mathrm{b}}$ School of Medical Science and Griffith Health Institute, Griffith University, Gold Coast QLD 4222, Australia.

Email addresses: 1.kidd@uq.edu.au; s4032723@ student.uq.edu.au; andy.wu@griffith.edu.au;

w.kelly@griffith.edu.au; $\underline{\text { m.forwood@griffith.edu.au }}$

Number of words: 3630

Number of Figures: 4

Number of Tables: 2

Running title: NSAIDs slow stress fracture repair

Funding sources: National Health and Medical Research Council Project Grants 401553,

511187; Rebecca L. Cooper Medical Research Foundation.

Address for Correspondence:

Professor Mark R Forwood

School of Medical Science

Griffith University Qld 4222

Australia

Tel +61 755527305

Fax +61 755528908

Email:m.forwood@griffith.edu.au 


\begin{abstract}
Anti-inflammatory drugs are widely used to manage pain associated with stress fractures, but little is known about their effects on healing of those injuries. We hypothesised that selective and non-selective anti-inflammatory treatments would retard the healing of stress fracture in the rat ulna. Stress fractures were created by cyclic loading of the ulna in Wistar rats. Ulnae were harvested 2, 4 or 6 weeks following loading. Rats were treated with non-selective NSAID, Ibuprofen $(30 \mathrm{mg} / \mathrm{kg} / \mathrm{d})$; selective COX-2 inhibition, DFU $(2.0 \mathrm{mg} / \mathrm{kg} / \mathrm{d})$; or the novel c5a anatagonist PMX53 (10 mg/kg/d, 4 and 6 weeks only); with appropriate vehicle as control. Quantitative histomorphometric measurements of stress fracture healing were undertaken. Treatment with the selective COX-2 inhibitor, DFU, reduced the area of resorption along the fracture line at 2 weeks, without affecting bone formation at later stages. Treatment with the nonselective, NSAID, ibuprofen decreased both bone resorption and bone formation so that there was significantly reduced length and area of remodelling and lamellar bone formation within the remodelling unit at 6 weeks after fracture. The C5a receptor antagonist PMX53 had no effect on stress fracture healing at 4 or 6 weeks after loading, suggesting that PMX53 would not delay stress fracture healing. Both selective COX-2 inhibitors and non-selective NSAIDs have the potential to compromise stress fracture healing, and should be used with caution when stress fracture is diagnosed or suspected.
\end{abstract}

\title{
Introduction
}

Non-steroidal anti-inflammatory drugs (NSAIDs), available as "over-the-counter" and prescription medications, are widely used to manage pain associated with musculoskeletal injuries ${ }^{1}$. NSAIDs act by inhibiting the effects of cyclooxygenase (COX) enzymes. COX-1 and COX-2 are the rate limiting enzymes involved in the conversion of arachidonic acid into prostaglandins. $\mathrm{PGE}_{2}$, primarily produced by $\mathrm{COX}-2$ during inflammation, has numerous roles in bone biology. $\mathrm{PGE}_{2}$ is a potent stimulator of osteoclastogenesis, and COX-2 and $\mathrm{PGE}_{2}$ are required for the response to many hormonal and cytokine mediators of bone resorption ${ }^{2}$. COX-2 and $\mathrm{PGE}_{2}$ are also important mediators of mechanotransduction, bone formation and bone adaptation to loading ${ }^{3}$.

There is convincing evidence that selective COX-2 inhibitors and non-selective NSAIDs compromise the healing of complete fractures ${ }^{4-8}$. Yet, very little is known about the effect of NSAIDs on stress fracture (SFx) healing ${ }^{9}$. Stress fractures are a common injury affecting children, adolescents and adults participating at all levels of sport and other athletic endeavours 
10-12. These injuries are frustrating and debilitating, often incurring considerable time lost from exercising, rehearsal or military training ${ }^{13,14}$. Stress fractures are characterised by incomplete fractures that develop as a result of repetitive loading on a bone rather than a single traumatic event. Some "high risk" SFx are prone to delayed healing or catastrophic propagation to complete fractures.

Repetitive loading of the rat ulnar creates an excellent model of SFx. It is non-invasive, avoids external trauma to the periosteum and its clinical characteristics are very similar to SFx in humans and animals ${ }^{15,16}$. In addition, the model is sensitive to pharmaceutical treatments that may influence resorption or formation phases of SFx healing ${ }^{9,17}$. Because of their wide availability and use, it is important to examine the effects non-selective NSAIDs on the healing of SFx. In animal models, the non-selective NSAIDs cause a greater negative effect on fracture healing ${ }^{18,19}$. Stress fractures created by ulnar loading heal by a combination of woven bone formation and remodelling that progresses directly along the fracture line ${ }^{16}$. Even after 10 weeks, there are regions of the fracture that have not completely healed, indicating that SFx healing is slower than the healing of complete fracture. In addition, SFx repair does not rely on endochondral ossification for fracture healing 9, 16, 17. COX-2 inhibition may, therefore, have different effects to that seen in complete fracture healing.

$\mathrm{C} 5 \mathrm{a}$ is a protein fragment, termed an anaphylatoxin, released from complement component $\mathrm{C} 5$ during activation of the complement system ${ }^{20}$. It is a potent inflammatory mediator and is involved in the progression of immuno-inflammatory conditions such as rheumatoid arthritis, inflammatory bowel disease, asthma and psoriasis ${ }^{21}$. A C5a receptor antagonist, PMX53, has been trialled as a NSAID, demonstrating potent inhibition of C5a mediated neutrophil chemotaxis, phagocytosis and leukocyte release of inflammatory cytokines ${ }^{22,23}$. Is has been used to target the complement system and has shown positive therapeutic effects in animal models of inflammatory arthritis ${ }^{24}$. Through its actions on inflammation and production of inflammatory cytokines, this new class of drug may prove to have indications in other musculoskeletal conditions. PMX53 treatment was more effective than ibuprofen in reducing joint swelling, joint cell numbers, and intra-articular TNFa and IL-6. TNFa and IL-6 are also important mediators of osteoclastogenesis ${ }^{25}$. As a novel anti-inflammatory agent, it is important to establish that a c5a inhibitor does not have detrimental effects on bone healing.

The first aim of this study was to determine the effect of the anti-inflammatory drugs on SFx healing and woven bone consolidation in the rat ulna. Our hypothesise is that treatment with the COX-2 inhibitor DFU and ibuprofen will delay remodelling of the fracture line and subsequent 
consolidation of periosteal woven bone, but will not affect woven bone production and that DFU will have a more pronounced effect than ibuprofen. Our second aim is to investigate the effect of PMX53 on SFx healing and woven bone consolidation. We hypothesise that treatment with a novel anti-inflammatory C5A antagonist will delay remodelling of the fracture line and consolidation of periosteal woven bone but will not affect woven bone production.

\section{Materials and methods}

\section{Loading model}

The University of Queensland Animal Ethics Committee approved all treatments. Loading sessions were conducted by placing the flexed carpus and elbow in cups and applying axial cyclic loading on the ulnae of rats using methods we described previously ${ }^{16,17}$. Briefly, displacement in the limb during loading was monitored using a LVDT connected to the loading device, and recorded using Maclab (AD Instruments, Colorado Springs, Colorado). Under isoflourane and oxygen anesthesia, loading was applied as cyclic compressive loading at 17-24 N load and $2 \mathrm{~Hz}$ cycle frequency. Loading was manually stopped at the point when a $10 \%$ increase in displacement was reached, compared to the lowest measured displacement. The total number of cycles to fatigue ranged from 3124 to 16480 cycles. A single injection of an opioid analgesic (Buprenorphine $0.05 \mathrm{mg} / \mathrm{Kg}$ subcutaneously) was used following loading sessions. No evidence of lameness or behavioural changes was seen following loading, nor was food or water intake different among groups.

\section{Treatment groups}

\section{Selective COX-2 inhibitor (DFU) treatment}

The right ulna was loaded in 60 rats. Drug treatment was commenced immediately after loading. For each timepoint rats were either treated with vehicle, or selective COX-2 inhibitor DFU at 2.0 $\mathrm{mg} / \mathrm{kg} /$ day. DFU ([5,5-dimethyl-3-3 (3 fluorophenyl)-4-(4 methylsulphonal) phenyl-2 (5H)furanone], Merck and Co, USA) has at least 1000 fold selectivity for COX-2 over COX-1 ${ }^{26}$. DFU binds selectively to the active site of COX-2 in a manner that appears to be competitive with arachidonic acid ${ }^{27}$. DFU was made up as a $2 \mathrm{mg} / \mathrm{ml}$ solution in the vehicle, polyethylene glycol (PEG). Rats were weighed daily to ensure the correct administration of the dose. Each rat received either DFU or the same volume of PEG by oral gavage daily until the end of the experiment. Rats were euthanised at 2, 4, or 6 weeks after loading ( $\mathrm{n}=10$ rats per group).

\section{Ibuprofen treatment}

The right ulna was loaded in 80 rats. Drug treatment was commenced immediately after loading. Rats were euthanised at 2, 4, or 6 weeks after loading. There were 10 rats in each group at 2 
weeks and 15 rats in each group at 4 and 6 weeks. For each timepoint rats were treated with ibuprofen at $30 \mathrm{mg} / \mathrm{kg} / \mathrm{d}$ or vehicle (distilled water) alone. Drugs were made up as a solution in distilled water, provided as the only drinking water until the end of the experiment. Drinking water was measured to ensure the correct administration of the dose.

\section{C5a receptor antagonist (PMX53) treatment}

The right ulna was loaded in 30 rats. This comprised 2 groups of rats treated with the C5a antagonist PMX-53 at $10 \mathrm{mg} / \mathrm{kg} / \mathrm{d}$ and euthanized at 4 and 6 weeks after loading $(\mathrm{n}=15$ per group). Due to a limited supply of PMX53, groups were not included at 2 weeks post-loading. Drugs were made up as a solution in distilled water, provided as the only drinking water until the end of the experiment. The same control animals were used for both the ibuprofen and PMX53 groups. Drinking water was measured to ensure the correct administration of the dose.

\section{Sample attrition}

There were 2 complete fractures during loading and these animals were euthanised immediately. Three complete fractures occurred during post-mortem dissection. Ten more samples were lost due to problems arising during tissue processing. The final numbers analysed for histomorphometry are given in Error! Reference source not found. and Error! Reference source not found. 2 .

\section{Histomorphometry}

Following euthanasia, a $10-15 \mathrm{~mm}$ section of the right ulna containing the fracture was removed from the bone. Fixed bones were decalcified in EDTA before being embedded in paraffin using standard protocols. One reader analyzed the sections and was blinded to group affiliation. As previously described ${ }^{17}$, histomorphometry was performed on one toluidine blue-stained section from each bone at a standard level along the fracture. This section was the point where the fracture line was half-way between the medial cortical margin and the medullary cavity of the bone in transverse section. Areas of woven bone, areas of osteoclastic resorption and cement lines between the original and newly formed intracortical bone could be clearly distinguished and measured. Histomorphometry measurements were obtained using a digitizing pad (Wacom Intuo 2, Vancouver, WA) and KSS software (KSS Stereology, Salt Lake City, UT). Measurements were made directly from histology slides using a digitizing pad connected to an Olympus BX60 microscope. Cortical and woven bone measurements obtained were cortical area (Ct.Ar, mm2) and woven bone area (Wo.B.Ar, mm2). Woven bone area was also normalized as a percentage of cortical bone area (Wo.B.Ar/Ct.Ar \%). 
Measurements along the fracture line, as shown previously ${ }^{17}$, included total length of the SFx (crack) $($ Cr.Le, $\mu \mathrm{m})$, total length of repair along the crack (Re.Cr.Le, $\mu \mathrm{m})$ and length of new bone formation along the crack (Ne.B.Cr.Le, $\mu \mathrm{m})$. The length of repair of the crack included regions that had previously been resorbed by osteoclasts, with or without newly formed bone within the BMU. The percentage resorption along the crack (Re.Cr.Le/Cr.Le, \%) and percentage of new bone along the crack (Ne.B.Cr.Le/Cr.Le) were normalized to correct for variations in the total length of the fracture line. Intracortical measurements obtained were total area of intracortical resorption (Rs.Ar, $\mu \mathrm{m} 2$ ), and intracortical area of healed new bone (Ne.B.Ar, $\mu \mathrm{m} 2)$. The areas of resorption and new bone formation were normalized by cortical area (Rs.Ar/Ct.Ar, \% and Ne.B.Ar/Ct.Ar, \%). We have previously determined that resorption area and length along the stress fracture line characterise resorptive activity in the model, hence osteoclast numbers were not counted ${ }^{16,17}$.

Mechanical testing was not performed in this study. In this model, woven bone formation restores whole-bone mechanical strength within 12 days of loading, well before stress fracture healing is complete $^{28,29}$. Mechanical testing would only reflect major changes in woven bone formation that are independent of the remodelling process along the fracture line ${ }^{15,16}$. Mechanical testing of whole bones is therefore not usually used to evaluate crack healing and remodelling along the stress fracture in this model ${ }^{9,16,17,30}$.

\section{Statistical analysis}

All variables were determined to have a normal distribution using a Kolmogorov-Smirnov test. Data were analysed using a two-way ANOVA. If this revealed a significant treatment effect differences between groups were determined using a one-way ANOVA with a post hoc Fisher's protected least significant difference. For all tests, significance was determined at $\mathrm{P}<0.05$.

\section{Results}

\section{Stress fracture healing}

All loaded bones developed SFxs. These were consistently in the distal half of the diaphysis on the medial (compressive) surface of the bone. There were no significant differences between any of the groups for fracture length, cortical area or bone width indicating that there was a consistent level of damage at a standard position within the bone. The configuration and qualitative assessment of the remodelling, healing and woven bone responses in all groups were very similar to those described for this model previously ${ }^{16,17}$. 


\section{Histomorphometry}

Woven bone formation: Results for cortical and woven bone histomorphometry are summarized in Tables 1 and 2. Woven bone area normalized by cortical area (WoB.Ar/Ct.Ar) was rapidly produced in the first 2 weeks after loading. This woven bone area was greatest at 2 weeks. Woven bone area was decreased at 4 weeks and remained stable between 4 and 6 weeks. There were no significant differences in $\mathrm{WoB}$.Ar/Ct.Ar between treated and control groups at any timepoint for Ibuprofen, PMX53 or DFU treatment.

Remodeling along the Fracture Line: At 2 weeks there was active remodelling along the fracture line, primarily in the region of the fracture where it exited the cortex. Remodelling was in the form of large resorption spaces that were progressing along the fracture towards the medullary region of the cortex. These resorption spaces had a crenated edge and were almost completely filled with cellular activity including obvious multinucleated osteoclasts. There was rarely new bone within the resorption spaces at this time-point (Figure 1).

There was a rapid increase in resorption area and healed new bone between 2 and 4 weeks after loading. By 4 weeks, more of the resorption space was occupied by new bone formation than by bone resorption. Six weeks after loading, individual resorption spaces could be seen and these had progressed further along the fracture line towards the medullary cavity.

NSAID treatment: Ibuprofen significantly reduced bone resorption and formation within the BMUs 6 weeks after loading, and DFU significantly reduced resorption along the fracture line 2 weeks after loading.

In the DFU-treated group, the normalized area of intracortical resorption spaces (Rs.Ar/Ct.Ar \%) was significantly less than in controls at 2 weeks $(p<0.05)$ (Figure 2). There were no significant differences between groups treated with ibuprofen and control groups at 2 weeks. There were no significant differences between DFU, Ibuprofen or PMX53-treated groups and control groups at 4 weeks for any resorption or new bone formation measurements.

At 6 weeks, the percentage of crack length occupied by new bone (Ne.B.Cr.Le/Cr.Le \%), the percentage of crack undergoing repair (Re.Cr.Le/Cr.Le \%), the normalized area of intracortical resorption spaces (Rs.Ar/Ct.Ar \%), and the normalized area of intracortical new bone (Ne.B.Ar/Ct.Ar) were all significantly reduced in the ibuprofen-treated group compared to controls goup $(p<0.05)$ (Figure 3 and Figure 4). There were no significant differences between DFU or PMX53 treated groups and control groups at the 6 week time-point. 


\section{Discussion}

Although the effect of NSAIDs on SFx healing is largely unknown, they are commonly used for analgesia. We showed a negative effect of a selective COX-2 inhibitor (DFU) and a non-selective NSAID (Ibuprofen) on SFx healing. This negative effect was due to reduction in both bone resorption and bone formation during the remodelling phases responsible for repairing the SFx line. In addition, administration of a novel C5a receptor antagonist (PMX53) had no significant effect on bone resorption or formation in this model.

Fracture union, mechanical properties, histological grading and callus composition of complete

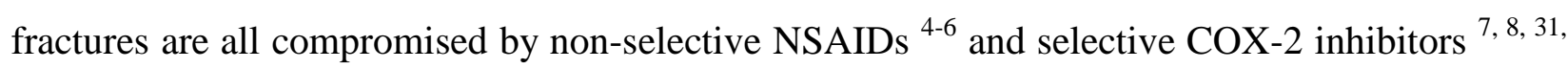
32 . Complete fractures heal by formation of fibrous tissue, cartilage callus and endochondral ossification; involving overlapping stages of tissue inflammation, repair and remodelling. COX-2 inhibitors affect aspects of all of these processes. $\mathrm{PGE}_{2}$ is important in osteoblastogenesis and COX-2 inhibitors reduce measures of bone formation in vitro ${ }^{33-35}$. $\mathrm{PGE}_{2}$ stimulates BMP-2 production by osteoblast progenitor cells ${ }^{36}$ and COX-2 is required for both endochondral ossification and woven bone formation during fracture repair ${ }^{34,37}$. Our data show that COX inhibitors also impair healing of SFxs. In contrast to complete fractures, direct remodelling repairs the SFx. Large resorption spaces progress along the fracture line and are eventually filled with new bone ${ }^{16}$. In the current study the area of resorption along the fracture line was significantly reduced by DFU at 2 weeks and by ibuprofen at 6 weeks after fracture.

COX-2 is important in states of generalised activated remodelling ${ }^{38,39}$, and promotes bone loss in inflammatory conditions by mediating osteoblastic RANKL production that increases osteoclastogenesis ${ }^{40-42}$. Our data clearly indicate that COX-2 is also important in resorption associated with focal, directed remodelling in response to fatigue injury. Ibuprofen also inhibited lamellar bone formation within the resorption spaces along the fracture line. Celecoxib also reduced bone formation in the rat ulnar, 8 weeks after SFx ( $\mathrm{Li}$ et al 2007). The vital role of COX2 in bone formation is also demonstrated in several experimental models $34,43,44$. Both nonselective and selective COX-2 inhibitors also reduce mechanically-induced lamellar bone formation when given prior to mechanical loading ${ }^{3}$. In these experiments, selective COX-2 inhibitors demonstrate a greater effect ${ }^{3,45}$.

Significant decreases in lamellar bone area with ibuprofen treatment were not seen until 6 weeks after loading. The timing of this effect may be a function of the time required to achieve an area of new bone formation great enough to detect significant changes. It could also be due to an indirect effect of the decreased osteoclast activity within resorption spaces. Resorption space area 
and length along the crack were significantly reduced by ibuprofen at 6 weeks. Osteoblast function and bone formation are tightly balanced with the activity of osteoclasts during remodelling. The exact factors involved in this "coupling" are not known but proposed mechanisms include release of growth factors from bone matrix, mediators ("coupling factors") released from osteoclasts, changes in the local strain environment, TRAP signalling and surface contour effects ${ }^{46-49}$. Osteoblast activity and bone formation may therefore have been affected by inhibition in osteoclast activity with reduced remodelling along the SFx line.

In this study ibuprofen had a more pronounced effect on stress fracture healing than DFU. While DFU significantly reduced the area of resorption along the fracture line 2 weeks after loading, ibuprofen significantly reduced the length and area of the BMUs and new bone formation 6 weeks after loading. This is a somewhat surprising given a hypothesis that specific COX-2 inhibition would have a greater effect on healing. The overwhelming evidence from in vitro and in vivo studies is that COX-2 is primarily responsible for the production of $\mathrm{PGE}_{2}$ in response to mechanical loading, fracture repair and bone resorption 3, 34, 50-52. However, COX-1 gene expression was significantly elevated in the ulna at 4 days after stress fracture creation, and this was at the same time as a significant increase in the expression of COX-2 ${ }^{16}$. It is possible that COX-1 contributes to the maximal production of $\mathrm{PGE}_{2}$ or other prostaglandins required during fracture healing. Inhibition of both $\mathrm{COX}-1$ and $\mathrm{COX}-2$ could then explain the more severe effect of ibuprofen on healing of the stress fracture. This enhanced reduction in healing parameters following administration of non-selective NSAIDs, compared to selective COX-2 inhibition, is not a unique observation $18,19,53$.

A limitation of this study was that the DFU and ibuprofen groups were administered using different protocols. Ibuprofen and PMX53 and were administered in the daily drinking water. Because the DFU was not soluble it was mixed into a suspension in PEG and the DFU and corresponding vehicle control groups were dosed daily by oral gavage. Serum ibuprofen levels were therefore likely to have been maintained throughout the day while DFU may have had peaks and troughs in the serum levels of the drug. The negative effects of COX-2 inhibition are reversible in complete fractures in the rat, and this appears to be due to a "rebound" effect of increased levels of $\mathrm{PGE}_{2}$ after withdrawal of medication ${ }^{54}$. At 10 times the oral dose used in this study, DFU plasma concentrations in rats peak at 1-2 hours and decline by 8 hours ${ }^{27}$. In the current study, treatment with DFU led to a significant reduction in bone resorption at 2 weeks, but resorption and formation were able to proceed sufficiently after that timepoint to prevent any significant differences in fracture healing at 4 and 6 weeks. Very small rebounds in $\mathrm{PGE}_{2}$ levels 
each day may account for some of the difference between ibuprofen and DFU administration. However the $\mathrm{ED}(50)$ for DFU in inflammatory models in the rat is $1 \mathrm{mg} / \mathrm{kg}^{26}$ and the dose used in this study $(2 \mathrm{mg} / \mathrm{kg})$ significantly reduces trabecular bone loss following ovariectomy (OVX) in the rat ${ }^{39}$.

Consistent with earlier reports, neither ibuprofen, nor DFU, reduced woven bone area or the maximum width of woven bone at any timepoint after loading. For example, increases in mechanically-induced endocortical lamellar bone formation in the rat tibia was reduced by indomethacin, and prevented by COX-2 inhibition (NS-398); but periosteal woven bone was not affected by either drug ${ }^{3}$. Moreover, daily administration of the selective COX-2 inhibitor (DFU) delayed the woven bone response to tibial 4-point bending but it did not reduce the peak woven bone area ${ }^{55}$. Similarly, woven bone formation following ulnar loading was not affected by celecoxib (Celebrex), while intracortical bone formation rate was reduced at 8 weeks after loading 9 . As the woven bone "callus" creates a rapid return to structural integrity of the bone, it is a critical component of the repair of fatigue damage and is likely to involve a robust cascade of cellular activity. Inhibition of COX-2 and $\mathrm{PGE}_{2}$ do not appear to be rate-limiting or magnitudelimiting components of this prolific woven bone response.

Treatment with the novel anti-inflammatory drug PMX53 was only studied at 4 and 6 weeks after SFx. At these timepoints, it did not affect any indices of bone resorption or formation during fracture healing. This suggests that this drug is unlikely to have a detrimental effect on bone healing. Although PMX53 reduces TNFa and IL-6 levels in a model of inflammatory arthritis ${ }^{24}$, inhibition of C5a function using PMX53 did not influence bone resorption in this study. This implies that $\mathrm{C} 5 \mathrm{a}$ is not part of the central pathway required for osteoclastogenesis during directed remodelling. PMX53 does reduce inflammatory hypernociception, however, and therefore has the potential to relieve inflammatory pain ${ }^{21}$. These data suggest the hypothesis that a c5a antagonist could provide necessary analgesia during (stress) fracture healing, without compromising the remodelling processes of bone healing.

In conclusion, treatment with the selective COX-2 inhibitor, DFU, reduced the area of resorption along the fracture line at 2 weeks, without affecting bone formation at later stages. Treatment with the non-selective, NSAID, ibuprofen decreased both bone resorption and bone formation so that there was significantly reduced length and area of remodelling and lamellar bone formation within the remodelling unit at 6 weeks after fracture. Ibuprofen reduced SFx remodelling to a greater extent than DFU, which may be due to differences in administration protocol between the two drugs. But we hypothesize that differences in potency against $\mathrm{COX}-2$, or a role for $\mathrm{COX}-1$ 
during remodelling could explain the difference. The C5a receptor antagonist PMX53 had no effect on SFx healing at 4 or 6 weeks after loading, suggesting that PMX53 would not delay SFx healing. Both selective COX-2 inhibitors and non-selective NSAIDs should be used with caution in clinical SFxs. Further study to determine the effects of NSAIDs in SFxs in humans and in racing and companion animals is warranted.

\section{Acknowledgments}

This study supported in part by National Health and Medical Research Council Project Grants 401553 and 511187; and the Rebecca L Cooper Medical Research Foundation. We thank Cephalon Australia (formerly Promics Pty Ltd) for the kind donation of PMX53; and, Merck and Co, USA for the kind donation of DFU. We acknowledge the technical assistance of Nurul Haji Mat Rasil.

\section{References}

1. Mehallo, CJ, Drezner, JA, Bytomski, JR. 2006 Practical management: nonsteroidal antiinflammatory drug (NSAID) use in athletic injuries. Clin J Sport Med 16: 170-174.

2. Li, L, Pettit, AR, Gregory, LS, Forwood, MR. 2006 Regulation of bone biology by prostaglandin endoperoxide H synthases (PGHS): a rose by any other name. Cytokine Growth Factor Rev 17: 203-216.

3. Forwood, MR. 1996 Inducible cyclo-oxygenase (COX-2) mediates the induction of bone formation by mechanical loading in vivo. J Bone Miner Res 11: 1688-1693.

4. Bo, J, Sudmann, E, Marton, PF. 1976 Effect of indomethacin on fracture healing in rats. Acta Orthop Scand 47: 588-599.

5. Allen, HL, Wase, A, Bear, WT. 1980 Indomethacin and aspirin: effect of nonsteroidal antiinflammatory agents on the rate of fracture repair in the rat. Acta Orthop Scand 51: 595-600.

6. Altman, RD, Latta, LL, Keer, R, et al. 1995 Effect of nonsteroidal antiinflammatory drugs on fracture healing: a laboratory study in rats. J Orthop Trauma 9: 392-400.

7. Endo, K, Sairyo, K, Komatsubara, S, et al. 2005 Cyclooxygenase-2 inhibitor delays fracture healing in rats. Acta Orthop 76: 470-474. 
8. Simon, AM, O'Connor, JP. 2007 Dose and time-dependent effects of cyclooxygenase-2 inhibition on fracture-healing. J Bone Joint Surg Am 89: 500-511.

9. Li, J, Waugh, LJ, Hui, SL, et al. 2007 Low-intensity pulsed ultrasound and nonsteroidal antiinflammatory drugs have opposing effects during stress fracture repair. J Orthop Res 25: $1559-1567$.

10. Bennell, KL, Brukner, PD. 1997 Epidemiology and site specificity of stress fractures. Clin Sports Med 16: 179-196.

11. Iwamoto, J, Takeda, T. 2003 Stress fractures in athletes: review of 196 cases. J Orthop Sci 8: 273-278.

12. Snyder, RA, Koester, MC, Dunn, WR. 2006 Epidemiology of stress fractures. Clin Sports Med 25: 37-52, viii.

13. Milgrom, C, Giladi, M, Stein, M, et al. 1985 Stress fractures in military recruits. A prospective study showing an unusually high incidence. J Bone Joint Surg Br 67: 732-735.

14. Raasch, WG, Hergan, DJ. 2006 Treatment of stress fractures: the fundamentals. Clin Sports Med 25: 29-36, vii.

15. Uthgenannt, BA, Silva, MJ. 2007 Use of the rat forelimb compression model to create discrete levels of bone damage in vivo. J Biomech 40: 317-324.

16. Kidd, LJ, Stephens, AS, Kuliwaba, JS, et al. 2010 Temporal pattern of gene expression and histology of stress fracture healing. Bone 46: 369-378.

17. Kidd, LJ, Cowling, NR, Wu, AC, et al. 2011 Bisphosphonate treatment delays stress fracture remodeling in the rat ulna. J Orthop Res 29: 1827-1833.

18. Gerstenfeld, LC, Thiede, M, Seibert, K, et al. 2003 Differential inhibition of fracture healing by non-selective and cyclooxygenase- 2 selective non-steroidal anti-inflammatory drugs. J Orthop Res 21: 670-675.

19. Brown, KM, Saunders, MM, Kirsch, T, et al. 2004 Effect of COX-2-specific inhibition on fracture-healing in the rat femur. J Bone Joint Surg Am 86-A: 116-123. 
20. Monk, PN, Scola, AM, Madala, P, Fairlie, DP. 2007 Function, structure and therapeutic potential of complement C5a receptors. Br J Pharmacol 152: 429-448.

21. Ting, E, Guerrero, AT, Cunha, TM, et al. 2008 Role of complement C5a in mechanical inflammatory hypernociception: potential use of C5a receptor antagonists to control inflammatory pain. Br J Pharmacol 153: 1043-1053.

22. Woodruff, TM, Strachan, AJ, Sanderson, SD, et al. 2001 Species dependence for binding of small molecule agonist and antagonists to the C5a receptor on polymorphonuclear leukocytes. Inflammation 25: 171-177.

23. Haynes, DR, Harkin, DG, Bignold, LP, et al. 2000 Inhibition of C5a-induced neutrophil chemotaxis and macrophage cytokine production in vitro by a new C5a receptor antagonist. Biochem Pharmacol 60: 729-733.

24. Woodruff, TM, Strachan, AJ, Dryburgh, N, et al. 2002 Antiarthritic activity of an orally active C5a receptor antagonist against antigen-induced monarticular arthritis in the rat. Arthritis Rheum 46: 2476-2485.

25. Cenci, S, Toraldo, G, Weitzmann, MN, et al. 2003 Estrogen deficiency induces bone loss by increasing $\mathrm{T}$ cell proliferation and lifespan through IFN-gamma-induced class II transactivator. Proc Natl Acad Sci U S A 100: 10405-10410.

26. Riendeau, D, Percival, MD, Boyce, S, et al. 1997 Biochemical and pharmacological profile of a tetrasubstituted furanone as a highly selective COX-2 inhibitor. Br J Pharmacol 121: 105117.

27. Nicoll-Griffith, DA, Falgueyret, JP, Silva, JM, et al. 1999 Oxidative bioactivation of the lactol prodrug of a lactone cyclooxygenase-2 inhibitor. Drug Metab Dispos 27: 403-409.

28. Hsieh, YF, Silva, MJ. 2002 In vivo fatigue loading of the rat ulna induces both bone formation and resorption and leads to time-related changes in bone mechanical properties and density. Journal of orthopaedic research : official publication of the Orthopaedic Research Society 20: 764-771. 
29. Silva, MJ, Touhey, DC. 2007 Bone formation after damaging in vivo fatigue loading results in recovery of whole-bone monotonic strength and increased fatigue life. Journal of orthopaedic research : official publication of the Orthopaedic Research Society 25: 252-261.

30. Sample, SJ, Hao, Z, Wilson, AP, Muir, P. 2011 Role of calcitonin gene-related peptide in bone repair after cyclic fatigue loading. PLoS One 6: e20386.

31. Endo, K, Sairyo, K, Komatsubara, S, et al. 2002 Cyclooxygenase-2 inhibitor inhibits the fracture healing. J Physiol Anthropol Appl Human Sci 21: 235-238.

32. Bergenstock, M, Min, W, Simon, AM, et al. 2005 A comparison between the effects of acetaminophen and celecoxib on bone fracture healing in rats. J Orthop Trauma 19: 717-723.

33. Evans, CE, Butcher, C. 2004 The influence on human osteoblasts in vitro of non-steroidal anti-inflammatory drugs which act on different cyclooxygenase enzymes. J Bone Joint Surg Br 86: 444-449.

34. Zhang, X, Schwarz, EM, Young, DA, et al. 2002 Cyclooxygenase-2 regulates mesenchymal cell differentiation into the osteoblast lineage and is critically involved in bone repair. J Clin Invest 109: 1405-1415.

35. Alander, CB, Raisz, LG. 2006 Effects of selective prostaglandins E2 receptor agonists on cultured calvarial murine osteoblastic cells. Prostaglandins Other Lipid Mediat 81: 178-183.

36. Arikawa, T, Omura, K, Morita, I. 2004 Regulation of bone morphogenetic protein-2 expression by endogenous prostaglandin E2 in human mesenchymal stem cells. J Cell Physiol 200: 400-406.

37. Simon, AM, Manigrasso, MB, O'Connor, JP. 2002 Cyclo-oxygenase 2 function is essential for bone fracture healing. J Bone Miner Res 17: 963-976.

38. Lane, N, Coble, T, Kimmel, DB. 1990 Effect of naproxen on cancellous bone in ovariectomized rats. J Bone Miner Res 5: 1029-1035. 
39. Gregory, LS, Kelly, WL, Reid, RC, et al. 2006 Inhibitors of cyclo-oxygenase-2 and secretory phospholipase A2 preserve bone architecture following ovariectomy in adult rats. Bone 39: 134-142.

40. Bukata, SV, Gelinas, J, Wei, X, et al. 2004 PGE2 and IL-6 production by fibroblasts in response to titanium wear debris particles is mediated through a Cox-2 dependent pathway. $\mathrm{J}$ Orthop Res 22: 6-12.

41. Lin, SK, Kok, SH, Kuo, MY, et al. 2002 Sequential expressions of MMP-1, TIMP-1, IL-6, and COX-2 genes in induced periapical lesions in rats. Eur J Oral Sci 110: 246-253.

42. Zhang, X, Morham, SG, Langenbach, R, et al. 2001 Evidence for a direct role of cyclooxygenase 2 in implant wear debris-induced osteolysis. J Bone Miner Res 16: 660-670.

43. Chikazu, D, Li, X, Kawaguchi, H, et al. 2002 Bone morphogenetic protein 2 induces cyclooxygenase 2 in osteoblasts via a Cbfal binding site: role in effects of bone morphogenetic protein 2 in vitro and in vivo. $\mathrm{J}$ Bone Miner Res 17: 1430-1440.

44. Rohde, C, Anderson, DE, Bertone, AL, Weisbrode, SE. 2000 Effects of phenylbutazone on bone activity and formation in horses. Am J Vet Res 61: 537-543.

45. Li, J, Burr, DB, Turner, CH. 2002 Suppression of prostaglandin synthesis with NS-398 has different effects on endocortical and periosteal bone formation induced by mechanical loading. Calcif Tissue Int 70: 320-329.

46. Hayden, JM, Mohan, S, Baylink, DJ. 1995 The insulin-like growth factor system and the coupling of formation to resorption. Bone 17: 93S-98S.

47. Gray, C, Boyde, A, Jones, SJ. 1996 Topographically induced bone formation in vitro: implications for bone implants and bone grafts. Bone 18: 115-123.

48. Smit, TH, Burger, EH, Huyghe, JM. 2002 A case for strain-induced fluid flow as a regulator of BMU-coupling and osteonal alignment. J Bone Miner Res 17: 2021-2029.

49. Martin, TJ, Sims, NA. 2005 Osteoclast-derived activity in the coupling of bone formation to resorption. Trends Mol Med 11: 76-81. 
50. Bakker, AD, Klein-Nulend, J, Burger, EH. 2003 Mechanotransduction in bone cells proceeds via activation of COX-2, but not COX-1. Biochem Biophys Res Commun 305: 677-683.

51. Okada, Y, Lorenzo, JA, Freeman, AM, et al. 2000 Prostaglandin G/H synthase-2 is required for maximal formation of osteoclast-like cells in culture. J Clin Invest 105: 823-832.

52. Okada, Y, Pilbeam, C, Raisz, L, Tanaka, Y. 2003 Role of cyclooxygenase-2 in bone resorption. J Uoeh 25: 185-195.

53. Karachalios, T, Boursinos, L, Poultsides, L, et al. 2007 The effects of the short-term administration of low therapeutic doses of anti-COX-2 agents on the healing of fractures. An experimental study in rabbits. J Bone Joint Surg Br 89: 1253-1260.

54. Gerstenfeld, LC, Al-Ghawas, M, Alkhiary, YM, et al. 2007 Selective and nonselective cyclooxygenase-2 inhibitors and experimental fracture-healing. Reversibility of effects after short-term treatment. J Bone Joint Surg Am 89: 114-125.

55. Gregory, LS, Forwood, MR. 2007 Cyclooxygenase-2 inhibition delays the attainment of peak woven bone formation following four-point bending in the rat. Calcif Tissue Int 80: 176-183.

\section{$\underline{\text { Figure Legends }}$}

Figure 1. Images demonstrate differences in detail from undecalcified ( $\sim 0$ um thickness) and decalcified (5 um thickness) sections (magnification 40X). A. Undecalcified, MMA embedded section $(87 \mu \mathrm{m})$ with resorption space (arrow) progressing along the stress fracture line (arrow head) at 2 weeks after loading. B. Thin section $(5 \mu \mathrm{m})$ stained with toluidine blue with similar resorption space at 2 weeks demonstrating clear cellular detail and distinction between resorption and new bone formation.

Figure 2. Effects of treatment with DFU or vehicle (control) on intracortical resorption space (Rs.Ar/Ct.Ar \%) at 2, 4 and 6 weeks after loading. Values are mean \pm SE for each group. ${ }^{a}$ $\mathrm{P}<0.05$ compared to control group same timepoint.

Figure 3. Effects of treatment with ibuprofen, PMX53 or VEH on the percentage of the crack length occupied by A. New bone (Ne.B.Cr.Le/Cr.Le\%) or B. repaired length along the crack 
(Re.Cr.Le/Cr.Le \%) at 2, 4 and 6 weeks after loading. Values are mean \pm SE for each group. ${ }^{\text {a }}$ $\mathrm{P}<0.05$ compared to control group at 6 weeks.

Figure 4. Effects of treatment with ibuprofen or PMX53 or vehicle (control) on the intracortical area occupied by A. new bone (Ne.B.Ar/Ct.Ar \%) or B. resorption space (Rs.Ar/Ct.Ar \%) at 2, 4 and 6 weeks after loading. Values are mean $\pm \mathrm{SE}$ for each group. ${ }^{\mathrm{a}} \mathrm{P}<0.05$ compared to control group at 6 weeks. 
Table 1 Cortical and woven bone histomorphometry results for ibuprofen and PMX53 treated groups

\begin{tabular}{|c|c|c|c|c|c|c|c|c|}
\hline \multirow{2}{*}{$\begin{array}{l}\text { Time-point } \\
\text { Treatment }\end{array}$} & \multicolumn{2}{|c|}{2 weeks } & \multicolumn{3}{|c|}{4 weeks } & \multicolumn{3}{|c|}{6 weeks } \\
\hline & $\begin{array}{l}\overline{\mathrm{VEH}} \\
(\mathrm{n}=10)\end{array}$ & $\begin{array}{l}\text { Ibuprofen } \\
(\mathrm{n}=9)\end{array}$ & $\begin{array}{l}\mathrm{VEH} \\
(n=13)\end{array}$ & $\begin{array}{l}\text { Ibuprofen } \\
(n=9)\end{array}$ & $\begin{array}{l}\text { PMX53 } \\
(n=10)\end{array}$ & $\begin{array}{l}\mathrm{VEH} \\
(n=14)\end{array}$ & $\begin{array}{l}\text { Ibuprofen } \\
(\mathrm{n}=15)\end{array}$ & $\begin{array}{l}\text { PMX53 } \\
(n=12)\end{array}$ \\
\hline Wo.B.Ar $\left(\mathrm{mm}^{2}\right)$ & $0.65(0.05)$ & $0.60(0.06)$ & $0.56(0.04)$ & $0.53(0.03)$ & $0.60(0.06)$ & $0.59(0.04)$ & $0.55(0.03)$ & $0.60(0.04)$ \\
\hline Wo.B.Ar/Ct.Ar \% & $53(6)$ & $48(4)$ & $48(5)$ & $41(2)$ & $51(4)$ & $46(5)$ & $42(2)$ & $48(2)$ \\
\hline
\end{tabular}

Values are mean (SEM) for cortical area (Ct.Ar), woven bone area (Wo.B.Ar) and woven bone area as a percentage of original cortical area (Wo.B.Ar/Ct.Ar \%) for daily treatment of vehicle (VEH), or ibuprofen (30mg/kg) or PMX53 (10 mg/kg) from rats at 2, 4 and $6 \mathrm{weeks}$ after loading.

There were no significant differences between treated and control groups at any time-point.

Table 2 Cortical and woven bone histomorphometry results for DFU treated groups

\begin{tabular}{|c|c|c|c|c|c|c|}
\hline Time-point & 2 weeks & & 4 weeks & & 6 weeks & \\
\hline Treatment & $\begin{array}{l}\mathrm{VEH} \\
(\mathrm{n}=10)\end{array}$ & $\begin{array}{l}\text { DFU } \\
(\mathrm{n}=10)\end{array}$ & $\begin{array}{l}\mathrm{VEH} \\
(\mathrm{n}=10)\end{array}$ & $\begin{array}{l}\text { DFU } \\
(n=9)\end{array}$ & $\begin{array}{l}\mathrm{VEH} \\
(\mathrm{n}=9)\end{array}$ & $\begin{array}{l}\text { DFU } \\
(n=10)\end{array}$ \\
\hline Wo.B.Ar $\left(\mathrm{mm}^{2}\right)$ & $0.72(0.07)$ & $0.69(0.06)$ & $0.61(0.03)$ & $0.72(0.04)$ & $0.65(0.04)$ & $0.59(0.04)$ \\
\hline Wo.B.Ar/Ct.Ar \% & $62(4)$ & $54(6)$ & $50(3)$ & $54(4)$ & $52(3)$ & $47(4)$ \\
\hline
\end{tabular}

Values are mean (SEM) for cortical area (Ct.Ar), woven bone area (Wo.B.Ar) and woven bone area as a percentage of original cortical area (Wo.B.Ar/Ct.Ar \%) for daily treatment of vehicle (VEH) or DFU $(2.0 \mathrm{mg} / \mathrm{kg})$-treated rats at 2, 4 and 6 weeks after loading. There were no significant differences between treated and control groups at any time-point. 